\title{
Intersubband resonances in InAs/AISb quantum wells: Selection rules, matrix elements, and the depolarization field
}

\author{
R. J. Warburton, C. Gauer, A. Wixforth, and J. P. Kotthaus \\ Sektion Physik der Ludwig-Maximilians-Universität München, Geschwister-Scholl-Platz 1, 80539 München, Germany \\ B. Brar and H. Kroemer \\ Department of Electrical and Computer Engineering, University of California, Santa Barbara, California 93106
}

(Received 2 May 1995)

\begin{abstract}
We present both theoretical and experimental results on the intersubband resonance in InAs/AlSb quantum wells. From a Kane $(\mathbf{k} \cdot \mathbf{p})$ description of the band structure we investigate the effect of the large nonparabolicity and of the high Fermi wave vector on the selection rules and matrix elements. The 1-2 transition in parallel excitation $(x)$ is shown to be very weak from simple parity arguments; in perpendicular excitation (z) the matrix element $\langle z\rangle$ is shown to be largely unaffected by nonparabolicity. The 1-3 transition turns out to be very weak in both geometries. Two band-gap engineering approaches to enhancing the parallel excitation of 1-2 are considered but the effect remains small as compared to the conventional $z$ excitation. In $z$ excitation the depolarization field condenses all the oscillator strength into essentially one sharp line despite the broadening expected from the nonparabolicity in the band dispersions. Inclusion of the depolarization field in the theory gives us good agreement with both the experimentally determined line shape and $\langle z\rangle$ matrix element.
\end{abstract}

\section{INTRODUCTION}

In the conventional picture of intersubband resonance in a quantum well it is argued that the confinement dictates that the electric vector of the light must lie along the growth direction $(z)$ in order to excite the transition. This is not ideal for potential device applications as a grating on the surface of the device is required to couple in normal incidence light through the near-field effect. This one-band picture of intersubband resonance has recently been challenged by several groups who consider a multiband description of the band structure. ${ }^{1-3}$ The general result is that through band coupling, notably coupling of the conduction states with the light-hole and heavy-hole states, all possible transitions are allowed, at least in principle, even in parallel $(x)$ excitation when the electric vector of the light lies in the plane of the quantum well. However, the detailed results are somewhat contradictory and confusing. Shik ${ }^{2}$ claims that the $1-2_{x}: 1-2_{z}$ intensity ratio approaches $1: 4$ in the limit of strong spin-orbit coupling. Conversely, Yang et al. ${ }^{1}$ maintain that in ordinary circumstances $1-2_{x}: 1-2_{z}$ is very small, but that it can be increased considerably by appropriate band-structure engineering. These authors also consider the 1-3 transition, where it is shown that the intensity of $1-3_{x}$ is larger than that of $1-3_{z}$, but no comparison is made with the strong $1-2_{z}$. Very recently, Peng and Fonstad ${ }^{3}$ suggested that coupling of the valence band to the higher conduction band leads to $1-2_{x}: 1-2_{z} \sim 1: 1$ for GaAs. There are also several experimental reports of strong intersubband absorption in parallel excitation. ${ }^{4-6}$ We attempt here to resolve some of these issues.

We present both calculations and experiments on InAs/ AlSb quantum wells, which are very well suited for an investigation of band coupling effects for a number of reasons. First, InAs has a small band gap so that the mixing of conduction-band $s$ and valence-band $p$ states is much larger than in the more conventional GaAs system. This mixing is responsible, for instance, for the large conduction-band nonparabolicity observed in this system. ${ }^{7}$ Secondly, the carrier densities can be very high, up to $5 \times 10^{12} \mathrm{~cm}^{-2}$, largely by virtue of the very high $(\sim 2 \mathrm{eV})$ conduction-band barrier height. This implies that the conduction band is occupied out to a very large in-plane wave vector $k_{\|}$, enhancing any matrix elements that depend on $k_{\|} \neq 0$. Finally, an InAs/AlSb quantum well that is symmetric under reflection can be considered to explore the consequences of parity. Of course, systems can be designed where the reflection symmetry is broken, as discussed below, and we find that this leads to results similar to those obtained more than a decade ago for InAs inversion layers.

Nonparabolicity has clearly a large influence on the subband energies, and it would therefore be expected that at high density the intersubband resonance would become broad as the 1-2 energy at $k_{\|}=0$ is substantially higher than that at the Fermi wave vector $k_{\|}=k_{f}$. However, we find that this broadening is eliminated by the depolarization field, the resonant screening by the high-density electron gas. In light of this, a second aim of the paper is to make some general comments as to the real role of band nonparabolicity in intersubband resonance.

\section{MATRIX ELEMENTS}

\section{A. Quantum well calculations}

In the envelope function scheme, the total wave function is expanded as

$$
\psi(\mathbf{r})=\sum_{i} \exp \left(i \mathbf{k}_{\|} \cdot \mathbf{r}\right) \phi_{i}(z)|i\rangle
$$


where the $|i\rangle$ are the Bloch functions, varying rapidly on the atomic scale, the $\phi_{i}(z)$ are the envelope functions, varying on the scale of the quantum well potential, and the factor $\exp \left(i \mathbf{k} \mathbf{k}_{\|} \cdot \mathbf{r}\right)$ simply expresses the translational symmetry in the well plane. The growth direction is taken as $z$ and in the following we consider just the $x$ direction in the plane. The $\phi_{i}$ 's are related to each other by an effective Hamiltonian, which can be derived in a number of different approximations. We are interested here in the properties of the conduction band so we have taken the Kane ${ }^{8}$ form of the Hamiltonian, which includes to $O\left(k^{2}\right)$ the couplings between the conduction, heavy hole, light hole, and spin-orbit split-off bands ( $s$, hh, lh, and so, respectively) but neglects the interactions with the other energetically remote bands. This model is known to reproduce at least the conduction-band nonparabolicity accurately, both for bulk InAs and InAs quantum wells, as can be judged, for instance, from cyclotron resonance. ${ }^{7}$ We do not reproduce all the details here, as they are straightforward elaborations of existing results, but rather comment on the salient points.

The Hamiltonian is $8 \times 8$ but nevertheless simple to solve following the observation of Yang et al. ${ }^{1}$ that the envelope functions for the conduction-band spin-up and spin-down states can be chosen independently. Physically, this means that the quantum well solutions are predominantly $s \uparrow$ or $s \downarrow$ but with admixtures of the $p$-like valence-band states. We use the spin, $\uparrow$ or $\downarrow$, as a label for the solutions, but it should be borne in mind that, because of the valence-band admixture, it is not a rigorous quantum number. The energy eigenvalues can be determined by setting one of the $s$-state $\phi$ 's to zero, and systematically eliminating all the other components. We define a position-dependent mass by

$$
\frac{1}{m^{*}(z)}=\frac{E_{p}}{3}\left(\frac{2}{E+\delta(z)}+\frac{1}{E+\Delta(z)+\delta(z)}\right),
$$

in direct analogy with the band-edge mass given by

$$
\frac{1}{m_{0}^{*}}=\frac{E_{p}}{3}\left(\frac{2}{E_{g}}+\frac{1}{E_{g}+\Delta}\right),
$$

where $E, E_{g}, \Delta, E_{p}$, and $\delta$ are the energy, the fundamental band gap, the spin-orbit splitting, the Kane energy, and the valence-band offset, respectively. The energy scale is defined such that the valence-band edge lies at energy $-\delta$ in each layer. $\delta$ is conveniently chosen to be zero for the well. The $\mathbf{k} \cdot \mathbf{p}$ Hamiltonian then yields

$$
\begin{aligned}
& \left(-\frac{\hbar^{2}}{2 m^{*}(z) m_{0}} k_{x}^{2}-k_{z} \frac{\hbar^{2}}{2 m^{*}(z) m_{0}} k_{z}+E_{g}(z)-\delta(z)\right) \phi_{1} \\
& \quad=E \phi_{1},
\end{aligned}
$$

$k_{z} \rightarrow-i \partial / \partial z$, and for a quantum well we have three separate layers where $E_{g}, \Delta$, and $\delta$ are constant, so that solutions can be found by solving Eq. (4) in the well and barriers and then by applying the boundary conditions ${ }^{9}$ that $\phi_{1}^{\text {well }}=\phi_{1}^{\text {barr }}$ and $1 / m_{\text {well }}^{*} \partial \phi_{1} / \partial z=1 / m_{\text {barr }}^{*} \partial \phi_{1} / \partial z$ at each interface. $m_{\text {well }}^{*}$ $\left(m_{\text {barr }}^{*}\right)$ is the mass obtained from Eq. (2) by inserting the well (barrier) parameters. Note that the nonparabolicity is revealed by the explicit energy dependence in all these equations.
TABLE I. The parameters used in the calculations for InAs/ $\mathrm{AlSb} / \mathrm{In}_{x} \mathrm{Ga}_{1-x} \mathrm{As}$ structures.

\begin{tabular}{lccc}
\hline \hline & $\mathrm{InAs}$ & $\mathrm{AlSb}$ & $\mathrm{In}_{x} \mathrm{Ga}_{1-x} \mathrm{As}$ \\
\hline$E_{g}(\mathrm{eV})$ & 0.418 & 2.32 & 0.677 \\
$\Delta(\mathrm{eV})$ & 0.38 & 0.75 & 0.371 \\
$E_{p}(\mathrm{eV})$ & 21.5 & & \\
$\delta(\mathrm{eV})$ & 0.0 & -0.198 & 0.059 \\
$\varepsilon_{r}$ & 12.25 & & \\
\hline \hline
\end{tabular}

The solutions for a $150-\AA$ InAs/AlSb quantum well, using the parameters as listed in Table I, are shown in Fig. 1. Although the barriers are very high the solutions differ drastically from the predictions of the square-well one-band model in that the energies are far from quadratically dependent on subband index, and in that the dispersions as a function of $k_{x}$ are obviously not parabolic even at moderate $k_{x}$.

To calculate the matrix elements between the states we make the standard separation of the $\mathbf{k} \cdot \mathbf{p}$ wave function in the electric dipole approximation, ${ }^{10}$

$$
\begin{aligned}
\left\langle\psi^{I}\left|k_{x(z)}\right| \psi^{F}\right\rangle= & \left(\sum_{i, j}\left\langle i\left|k_{x(z)}\right| j\right\rangle \int\left(\phi_{i}^{I}\right)^{*} \phi_{j}^{F} d z\right. \\
& \left.+\sum_{i} \int\left(\phi_{i}^{I}\right)^{*} k_{x(z)} \phi_{i}^{F} d z\right) \delta_{k_{x}^{I}, k_{x}^{F}}
\end{aligned}
$$

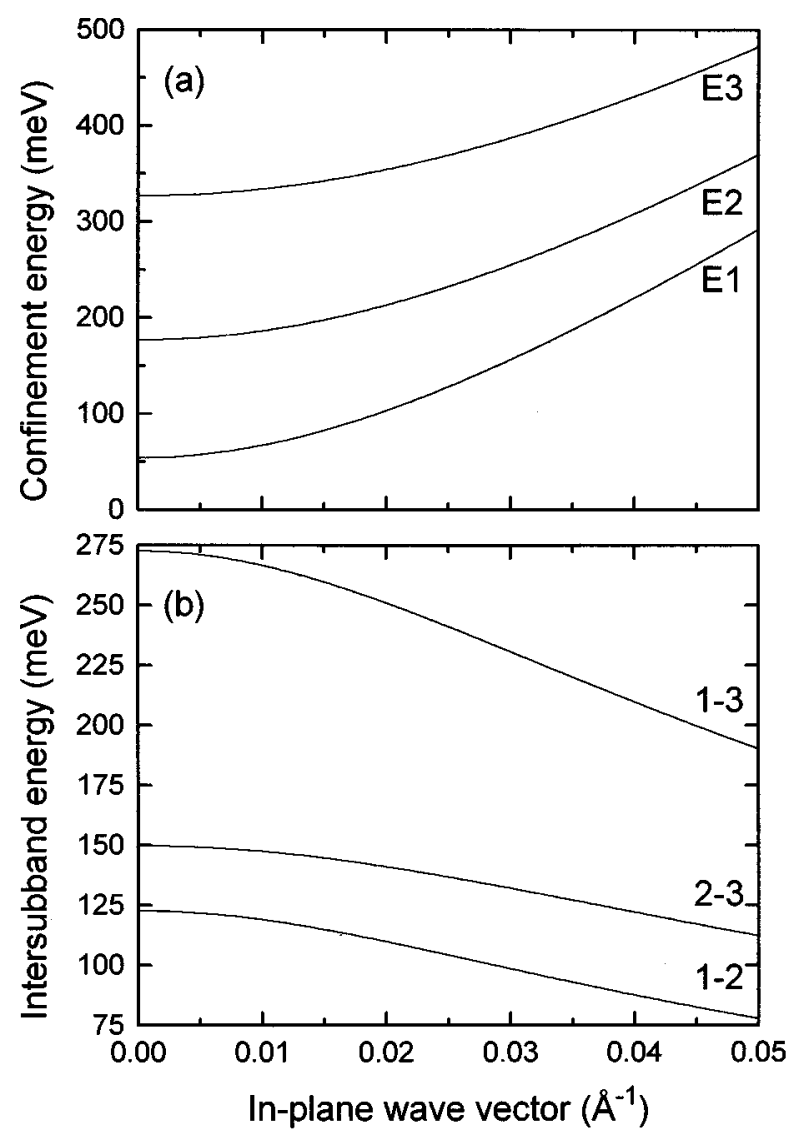

FIG. 1. (a) The subband confinement energies, and (b) the intersubband energies for a $150-\AA$ InAs/AlSb quantum well. 
TABLE II. The list of Bloch states in the Weiler representation. The parities of the envelope functions associated with each Bloch state are given for the $E 1 \uparrow$ and $E 1 \downarrow$ levels. The parities all reverse for the $E 2$ solutions.

\begin{tabular}{lccc}
\hline \hline State & Bloch & $E 1 \uparrow$ & $E 1 \downarrow$ \\
\hline 1 & $s \uparrow$ & even & 0 \\
2 & $\mathrm{hh} \uparrow$ & even & 0 \\
3 & $\mathrm{lh} \downarrow$ & even & odd \\
4 & $\mathrm{so} \downarrow$ & even & odd \\
5 & $s \downarrow$ & 0 & even \\
6 & $\mathrm{lh} \uparrow$ & odd & even \\
7 & $\mathrm{hh} \downarrow$ & 0 & even \\
8 & so $\uparrow$ & odd & even \\
\hline \hline
\end{tabular}

The first term is the "interband" term and dominates by a factor of $\sim 1 / m_{0}^{*}(\simeq 43$ for InAs) over the second "intraband" term, which can therefore be neglected for most practical purposes (although we have retained it in the numerical routines). To proceed we need to determine the nonzero $\left\langle i\left|k_{x(z)}\right| j\right\rangle$. We have adopted the labeling and phase conventions of Weiler ${ }^{11}$ (see Table II) and find that $s \uparrow$ couples through $k_{x}$ with $\mathrm{hh} \uparrow, \mathrm{lh} \downarrow$, and $\operatorname{so} \downarrow\left(\left\langle 1\left|k_{x}\right| 2\right\rangle,\left\langle 1\left|k_{x}\right| 3\right\rangle\right.$, $\left\langle 1\left|k_{x}\right| 4\right\rangle \neq 0$ ). Conversely, $s \uparrow$ couples through $k_{z}$ with the opposite spins, $\mathrm{lh} \uparrow$ and so $\uparrow$, but does not couple at all with the heavy-hole states. For $s \downarrow$, all the spins quite naturally reverse. In each case, these matrix elements are independent of $\mathbf{k}$ (as is obvious by writing a velocity operator as $\left.1 / \hbar \partial H / \partial k_{x(z)}\right),{ }^{12}$ differing one to another by small real numbers.

In the following we refer to $\left\langle\psi^{I}\left|k_{x(z)}\right| \psi^{J}\right\rangle$ by $M_{I J}^{x(z)}$, and $I-J_{x(z)}$ refers to the oscillator strength, which is $\propto\left|M_{I J}^{x(z)}\right|^{2}$. In every case transitions are allowed by interactions of the conduction-band component in one state with the valenceband components in the other. In this sense, intersubband transitions are not fundamentally different from interband transitions, as has been pointed out by Khurgin. ${ }^{13}$ With these results all that is necessary is to work out the corresponding overlap integrals of the envelope functions.

Some general remarks can be made simply from the symmetry under the transformation $z \rightarrow-z$ of the quantum well potential, which implies that each envelope function has a definite parity. The dominant component, for instance $\phi_{1}$ for spin-up, has even $(E 1, E 3$, etc.) or odd $(E 2, E 4$, etc.) parity, and the other components have the same parity if they are proportional to $k_{x} \phi_{1}$ (components 2, 3, and 4) or opposite parity if proportional to $k_{z} \phi_{1}$ (components 6 and 8). Similar remarks apply for the spin-down solutions. Simply from the parity we come to the selection rules listed in Table III,

TABLE III. The selection rules for intersubband transitions in a symmetric quantum well. $x(z)$ polarization implies that the electric vector lies perpendicular (parallel) to the growth direction.

\begin{tabular}{lcc}
\hline \hline Polarization & $1-2$ & $1-3$ \\
\hline$x$ & spin flip & spin conserving \\
$z$ & spin conserving & spin flip \\
\hline \hline
\end{tabular}

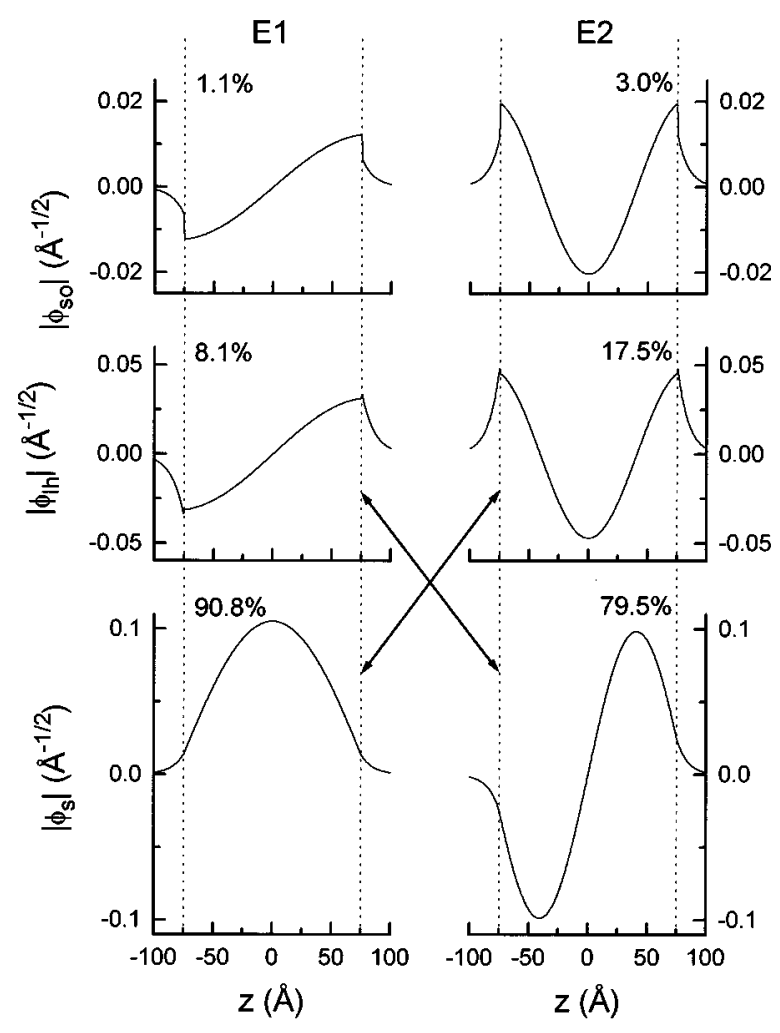

FIG. 2. The envelope functions $\phi_{i}$ relevant in the calculation of the electric dipole matrix elements for the transitions $1^{\uparrow}-2_{z}^{\uparrow}$ and $1^{\uparrow}-2 \downarrow$. The arrows show which $\phi_{s} \phi_{\mathrm{lh}}$ products must be integrated; the $s$ and so states are similarly connected. For $z(x)$ polarization, the $s, \mathrm{lh}$, and so states all have the same (opposite) spin. The percentages by each state are the amplitudes, defined by $\alpha_{i}=\int_{-\infty}^{\infty}\left|\phi_{i}\right|^{2} d z$.

which are in agreement with those of Yang et al. ${ }^{1}$ However, the question is, just how large are the matrix elements for the various transitions?

It is instructive to consider the simple but illustrative case at $k_{x}=0$, which we pursue analytically by making the very reasonable assumption that the vast majority of the wave function lies in the well. The envelope functions making up the $E 1$ and $E 2$ solutions are shown in Fig. 2, and the percentage marked by each state is a measure of the amplitude as defined by $\alpha_{i}=\int_{-\infty}^{\infty}\left|\phi_{i}\right|^{2} d z$. For instance, the $E 1 \uparrow$ state at $k_{x}=0$ is $90.8 \% s \uparrow$ like, $8.1 \% \mathrm{hh} \uparrow$ like, and $1.1 \%$ so $\uparrow$ like. The lh admixture increases to some $17.5 \%$ for the $E 2$ solution. The arrows in Fig. 2 show which $s$ and lh states couple according to the selection rules (Table III) and the nonzero $\left\langle i\left|k_{x(z)}\right| j\right\rangle$. For $k_{z}$ the four integrals for the spin-conserving process add and give a large matrix element, which can be calculated as

$$
\begin{gathered}
\frac{E_{p}}{6}\left[\frac{2}{E_{1}}+\frac{2}{E_{2}}+\frac{1}{E_{1}+\Delta}+\frac{1}{E_{2}+\Delta}\right] I \\
\quad=\frac{1}{2}\left[\frac{1}{m^{*}\left(E_{1}\right)}+\frac{1}{m^{*}\left(E_{2}\right)}\right] I
\end{gathered}
$$

with $I=\int\left(\phi_{1}^{E 1}\right) * k_{z} \phi_{2}^{E 2} d z$. This has the same form as the one-band result but the effective masses, $m^{*}\left(E_{1}\right)$ and $m^{*}\left(E_{2}\right)$, obtained by inserting $E_{1}$ and $E_{2}$ into Eq. (2), are 

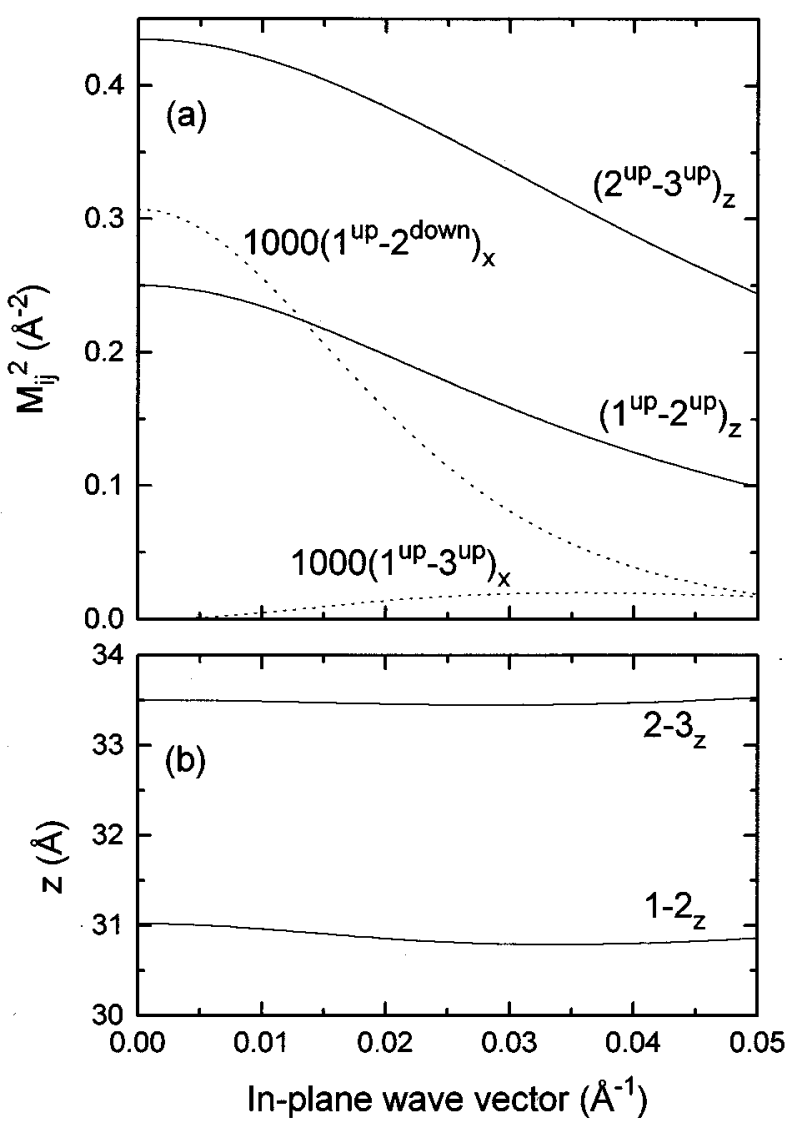

FIG. 3. (a) $\left|M_{I J}^{x(z)}\right|^{2}=\left|\left\langle\psi^{I}\left|k_{x(z)}\right| \psi^{J}\right\rangle\right|^{2}$ (z, solid lines; $x$, dotted lines), and (b) $\left\langle z_{I J}\right\rangle=\left|\left\langle\psi^{I}|z| \psi^{J}\right\rangle\right|$ as a function of the in-plane wave vector $k_{x}$ for a $150-\AA$ InAs/AlSb quantum well.

quite different from the band-edge value. For $k_{x}$, however, although each individual overlap integral for the $E 1-E 2$ transition is large, the signs are different so that the net matrix element is small. We find

$$
\frac{E_{p}}{6}\left[-\frac{1}{E_{1}}+\frac{1}{E_{2}}+\frac{1}{E_{1}+\Delta}-\frac{1}{E_{2}+\Delta}\right] I .
$$

The ratio $M_{12}^{x} / M_{12}^{z}$ is then small. In the limit that the confinement energies are small compared to the band gap, and writing $E_{2}-E_{1}=E_{12}$, we find

$$
\left|\frac{M_{12}^{x}}{M_{12}^{z}}\right| \simeq \frac{E_{12} \Delta\left(2 E_{g}+\Delta\right)}{2 E_{g}\left(E_{g}+\Delta\right)\left(3 E_{g}+2 \Delta\right)},
$$

which gives approximately $\left|M_{12}^{x}: M_{12}^{z}\right|^{2}=1-2_{x}: 1-2_{z} \simeq 0.1 \%$ for an InAs/AlSb quantum well with $E_{12} \simeq 100 \mathrm{meV}$. Note that when $\Delta=0$ the quantum interference is complete such that $M_{12}^{x}=0$; and also that an error in the phase can lead to the erroneous factor 1:4 in the limit $\Delta \rightarrow \infty$.

Exact numerical results for a $150-\AA$ quantum well are shown in Fig. 3. It can be seen that both $M_{12}^{x}$ and $M_{12}^{z}$ decrease with increasing $k_{x}$. The reason for this is that the nonzero $k_{x}$ causes other states to be admixed. For instance, the $E 1 \uparrow$ solution has considerable $\mathrm{hh} \uparrow$ and $\mathrm{lh} \downarrow$ amplitude for $k_{x} \neq 0$, yet these states do not contribute to the matrix element; the amplitude of the optically active states is then forced to diminish, leading to a reduction in the overall ma- trix element. This argument applies for both $M_{12}^{x}$ and $M_{12}^{z}$, but the numerical calculations of Fig. 3 show that the $k_{x}$-induced mixing is more deleterious for $M_{12}^{x}$ than for $M_{12}^{z}$. Figure 3 also shows the $M_{13}^{x}$ amplitude; the $M_{13}^{z}$ amplitude is not included because, even at $k_{x}=0.05 \AA^{-1}$, $M_{13}^{z}$ is as small as $2 \times 10^{-9} \AA^{-2}$. In both cases, $M_{13}=0$, when $k_{x}=0$ as the optically important light-hole states are mixed $\propto k_{x}$ in this case. For $M_{13}^{z}$ the parity forces the transition to be spin flip, and we find that the interference effect plays a role, as for $M_{12}^{x}$. This is not the case for the spinconserving $M_{13}^{x}$ where all the contributions add, but the matrix element is so small in this case because the overlap integrals themselves are small. We are thus in agreement with Yang et al. that $M_{13}^{x} \gg M_{13}^{z}$, but this is a somewhat specious comparison as $M_{13}^{x} \ll M_{12}^{z}$.

Figure 3 also shows the spatial matrix element $\left\langle z_{12}\right\rangle=\left|\left\langle\psi^{1}|z| \psi^{2}\right\rangle\right|$, which can be quite generally derived from

$$
\left\langle z_{12}\right\rangle=\frac{\hbar^{2}}{m_{0}} \frac{\left|M_{12}^{z}\right|}{E_{12}} .
$$

We find that $\left\langle z_{12}\right\rangle$ is essentially independent of $k_{x}$. This is despite the radical change in the wave-function admixture that occurs as $k_{x}$ is increased. Furthermore, the absolute value is very close to that obtained from a naive one-band model. In order to give a fair comparison with the multiband model, in the one-band calculation we take the energyindependent masses $m_{\text {well }}$ and $m_{\text {barr }}$ to be 0.023 and 0.117 , respectively, as given by Eq. (3) using the parameters of Table I, and assume continuity of $1 / m_{\text {well }} \partial \phi / \partial z$ $=1 / m_{\text {barr }} \partial \phi / \partial z$. We have, at $k_{x}=0,\left\langle z_{12}\right\rangle=31.0 \AA$ from the multiband model, and the very similar $33.2 \AA$ from the oneband model. The $\left\langle z_{23}\right\rangle$ matrix elements are also very similar, $33.5 \AA$ in the multiband calculation, as opposed to $34.8 \AA$ in the one-band calculation. The relative difference in $E_{12}$ (at $k_{x}=0$ ) between the calculations in the one-band model $(141.9 \mathrm{meV})$ and multiband model $(122.9 \mathrm{meV})$ is about the same as the relative difference in $\left\langle z_{12}\right\rangle^{2}$ for this $150-\AA$ quantum well. However, this no longer applies for a narrower quantum well where the $E_{12}$ energy is strongly influenced by the nonparabolicity, but the $\left\langle z_{12}\right\rangle$ matrix element remains the same to within $\sim 10 \%$ between the two models. We are thus led to conclude that nonparabolicity makes a significant difference to the intersubband energies but that spatial matrix elements are largely unaffected. This means that the selection rules and matrix elements as obtained from a naive approach are actually remarkably accurate.

\section{B. Alternative structures}

We have shown above that for an InAs/AlSb quantum well the matrix element $M_{12}^{x}$ is very small in comparison to $M_{12}^{z}$, largely for two reasons. First, the reflection symmetry forbids the spin-conserving transition in $x$ polarization, and secondly, for the allowed spin-flip transition the $E 1$ (lh)-E2 $(s)$ and $E 1(s)-E 2(\mathrm{lh})$ integrals tend to cancel. The purpose of this section is to examine to what extent these limitations can be overcome by considering a structure with no welldefined parity for which the spin-conserving $1-2_{x}$ transition 


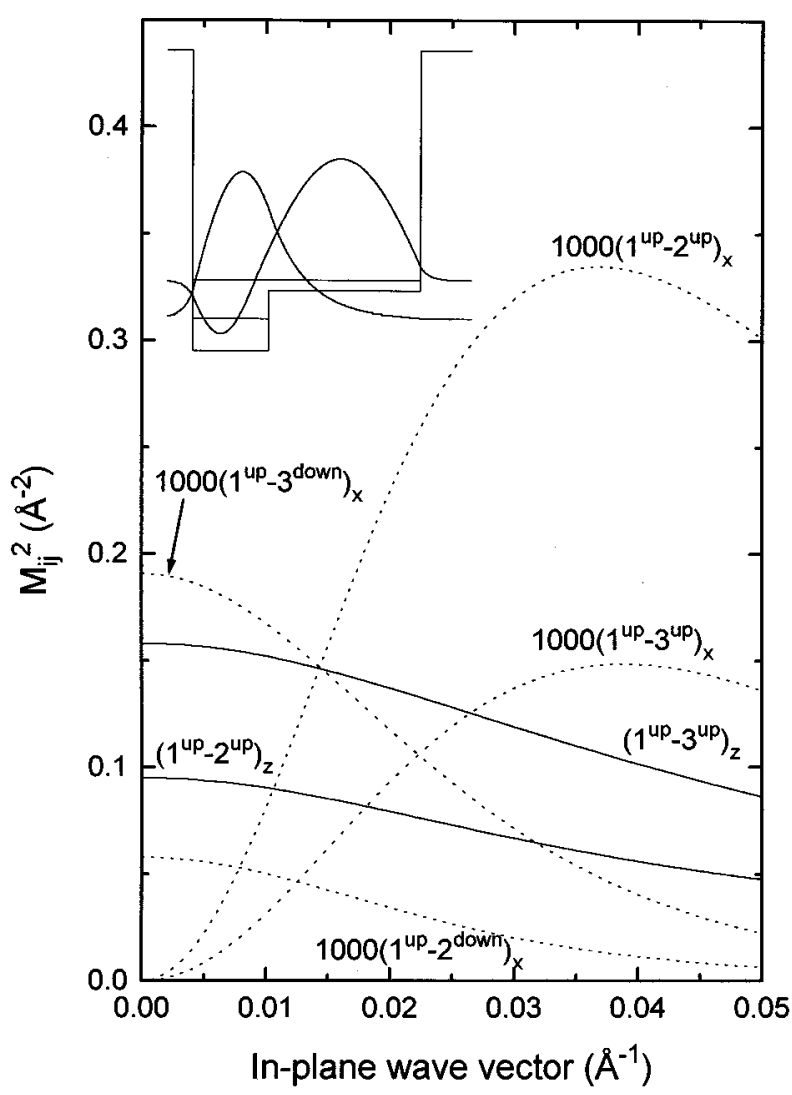

FIG. 4. $\left|M_{I J}^{x(z)}\right|^{2}$ for an asymmetric quantum well ( $z$, solid lines; $x$, dotted lines), the band lineup of which is shown in the inset along with the energies and $s$ components of the first two levels.

is no longer forbidden, and also a well where the light-hole coupling to $E 1$ is enhanced relative to that of $E 2$ so that the cancellation is far from complete.

In order to break the reflection symmetry we present calculations for the structure shown in the inset to Fig. 4, a quantum well with a step, in this case $\operatorname{InAs} / \mathrm{In}_{x} \mathrm{Ga}_{1-x} \mathrm{As}$. The parameters were chosen so that the first level $E 1$ is strongly confined to the InAs but the second level E2 lies above the energy of the step and so has a wave function that spreads over the entire structure. The $s$ envelope functions are sketched, and confirm that a parity cannot be defined. For a fair comparison with the straightforward InAs/AlSb quantum well, the widths and $\operatorname{In}_{x} \mathrm{Ga}_{1-x}$ As composition were chosen so that $E_{12}$ is to within a few $\mathrm{meV}$ the same as before. The momentum matrix elements are plotted as a function of $k_{x}$ in Fig. 4, and we make the following observations. $1^{\uparrow}$ $2_{z}^{\uparrow}$ is smaller than before, simply because of the lateral shift of the $E 2$ wave function. Conversely, $1^{\uparrow}-3 z_{z}^{\uparrow}$, which is forbidden by parity for a symmetric quantum well, is now large, and can be thought of approximately as the 1-2 transition in a wide well. The spin-flip $z$ transitions are, as before, exactly forbidden at $k_{x}=0$, and to all intents and purposes forbidden also when $k_{x} \neq 0.1^{\uparrow}-2_{x}^{\downarrow}$ behaves similarly to $1^{\uparrow}-2_{x}^{\downarrow}$ for the ordinary quantum well; namely, it is a factor of $\sim 1000$ smaller than $1^{\uparrow}-2_{z}^{\uparrow}$ at $k_{x}=0$ and decreases rapidly with increasing $k_{x}$. However, in contrast to the ordinary quantum well, $1^{\uparrow}-2_{x}^{\uparrow}$ is forbidden only at $k_{x}=0$ and increases quite strongly with $k_{x}$ up to $k_{x} \sim 0.03 \AA^{-1}$. The net contribution to
1-2 ${ }_{x}$ from both spin-flip and spin-conserving transitions, integrating out to $k_{x}=0.05 \AA^{-1}$, is approximately $0.4 \%$ of 1 $2_{z}$. However, although the $1-2_{x}$ intensity is increased relative to $1-2_{z}$ in this asymmetric structure, the maximum value attained by $1-2_{x}$ is actually comparable to that obtained in the ordinary quantum well case, which therefore means that there is no large increase in absolute oscillator strength. In other words, excitation with a parallel polarization remains weak.

The structure shown in Fig. 4 has eigenfunctions that are reasonably similar to those of InSb and InAs inversion layers, which were studied a decade ago. Our calculations are in broad agreement with those of this era. Takada and co-workers ${ }^{14}$ calculated the conductivities $\sigma_{x x}$ and $\sigma_{z z}$ for an InSb inversion layer, predicting that the optical intensity of $1-2_{x}$ is about two orders of magnitude smaller than $1-2_{z}$. Zawadzki, ${ }^{15}$ arguing as we do here from $s$ - $p$ hybridization, calculated a much larger ratio, of about $10 \%$, also for InSb. This latter theory omitted spin completely and reproduces neither the details of the spin-flip and spin-conserving transitions as described above nor the effects of spin-orbit interaction. Our own results predict factors of $\sim 1 \%$ for both $\mathrm{InSb}$ quantum wells and asymmetric InSb structures, which implies that even in the very narrow gap system $\mathrm{InSb}$ $\left(E_{g}=0.237 \mathrm{eV}\right)$ with strong spin-orbit interaction $(\Delta=0.8$ $\mathrm{eV}$ ), the parallel excitation mechanism is weak. The results of Takada and co-workers and Zawadzki had some experimental justification from optical experiments on both InSb (Refs. 16 and 17) and InAs (Ref. 18) inversion layers, where a doublet was observed in parallel excitation. It was thought that one resonance corresponds to $x$ and the other to $z$ excitation, assuming that surface roughness scrambles the polarization to some extent, and that the splitting arises because the depolarization field acts in the $z$ case, pushing the resonance to higher energy, but not in $x$. However, it was also noted that the splitting is very much what one expects from an additional spin-orbit splitting between the electron motion in the plane and the confining electric field in the growth direction. ${ }^{19}$ To the best of our knowledge, this controversy has never been completely resolved.

We have also considered a prototype system in InAs/ $\mathrm{AlSb} / \mathrm{Al}_{1-x} \mathrm{Ga}_{x} \mathrm{Sb}$, designed so that the $E 1$ level in the InAs well is confined by thin AlSb barriers but lies close to resonance with the valence-band edge in surrounding $\mathrm{Al}_{1-x} \mathrm{Ga}_{x} \mathrm{Sb}$ layers. This increases the $\mathrm{lh}$ admixture to the $E 1$ wave function. Conversely, the $E 2$ level at higher energy is not strongly affected. This structure has recently been proposed,${ }^{20}$ predominantly for a speculative intersubband laser application, as it might be possible to invert the lifetimes of $E 1$ and $E 2$. We present calculations for just 1-ML AlSb barriers with an outer barrier alloy concentration adjusted so that the $E 1$ level lies just $10 \mathrm{meV}$ above the $\mathrm{Al}_{1-x} \mathrm{Ga}_{x} \mathrm{Sb}$ band edge. Compared to an InAs/AlSb quantum well, $E_{12}$ and $\left|M_{12}^{z}\right|^{2}$ remain the same to within $\sim 10 \%$, but $\left|M_{12}^{x}\right|^{2}$ increases, by a factor of $\sim 1.9$ at $k_{x}=0$. The light-hole amplitude in the $E 1$ level also increases, from $8.1 \%$ to $16.4 \%$ for the new structure. Thus, it is possible to increase $M_{12}^{x}$ by increasing the light-hole amplitude of the $E 1$ wave function, but the effects are not particularly large. Furthermore, the enhancement rapidly disappears as the AlSb barriers are widened beyond $5 \AA$, and also the system must be designed so 


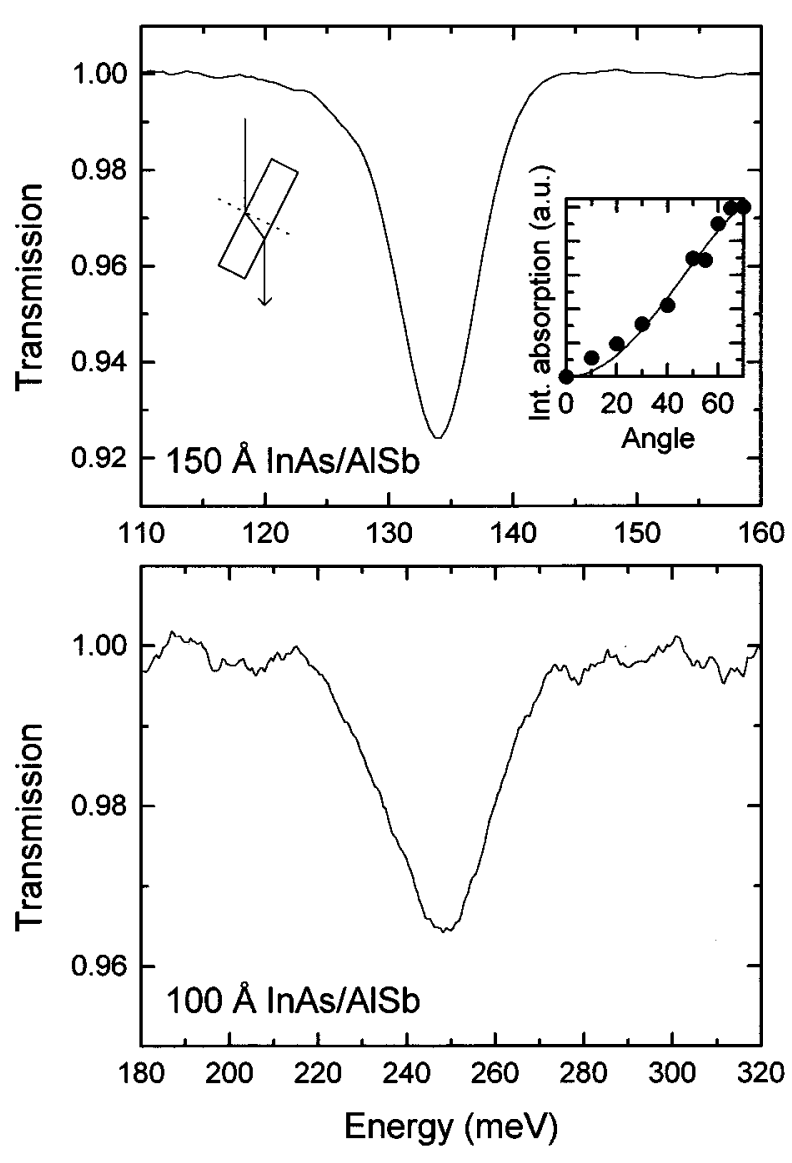

FIG. 5. Experimental spectra of InAs/AlSb multiple quantum wells taken in the Brewster angle geometry, as sketched. The inset shows the absorption intensity against angle to the normal (outside the sample) measured at room temperature; the solid line is $E_{z}^{2}$ $\propto \cos ^{2} \theta / \sin \theta$, where $\theta$ is the angle to the normal in the sample.

that the $E 1$ level is within only a few ten's of meV from resonance with the $\mathrm{Al}_{1-x} \mathrm{Ga}_{x} \mathrm{Sb}$ valence-band edge; conditions which would clearly be difficult to achieve in practice.

\section{Experiments}

The general conclusion from the theoretical considerations above on InAs/AlSb systems is that although parallel excitation of the intersubband transition is not completely forbidden it is very weak, even in specially designed structures. We have investigated this point experimentally with optical experiments on two InAs/AlSb multiple quantum well samples. The samples have 20 wells, of either 100 or $150 \AA$ thickness, carrier concentrations of $3.0 \times 10^{12}$ and $2.5 \times 10^{12} \mathrm{~cm}^{-2}$ per well, respectively, and were mounted into a 4-K cryostat. Spectroscopy was carried out with a Fourier-transform spectrometer with a reference spectrum taken either from a substrate or from another InAs/AlSb sample with different characteristics.

We carried out the experiments with the sample at various angles to the light beam. The data shown in Fig. 5 were taken with the samples at the Brewster angle and show strong intersubband absorption. With the sample exactly perpendicular to the light beam, and therefore with the polarization exactly in the plane of the quantum wells, we were unable to detect any absorption. This verifies that the strong absorption seen when the sample is tilted comes from the $E_{z}$ component. We checked this at room temperature (where we can polarize the incident light) by progressively tilting the sample. We eliminated the polarization, which is always parallel to the surface, and then measured the absorption intensity as a function of angle. The results are plotted in the inset to Fig. 5 and the solid line is $\cos ^{2} \theta / \sin \theta$, which is how $E_{z}$ depends on angle ${ }^{21}$ ( $\theta$ is the angle to the normal in the sample). The solid line reproduces the experimental results extremely well and therefore confirms that there is no strong $E_{x}$ excitation.

From our signal:noise ratio of $\sim 0.1 \%$ we can conclude experimentally that $1-2_{x}: 1-2_{z}<1 \%$, as anticipated from the above theory. (Note that the maximum absorption does not scale simply with the matrix elements, as we have a sharp line in $z$ excitation from the depolarization field, as explained below, but the full nonparabolicity-induced broadening in $x$ excitation, so making it more difficult experimentally to observe the $x$ transition.) We looked also for the 1-3 transition in both polarizations and likewise saw nothing, again consistent with the calculations. Although these results are perhaps not at all surprising, other systems ${ }^{4-6}$ have apparently given reasonably strong optical activity in parallel excitation. The explanation of these results is not obvious, particularly in light of the fact that the $1-2_{x}: 1-2_{z}$ ratio should decrease rapidly with increasing band gap. One possibility is that scattering, either from surface roughness or from microscopic defects or even from the edges of a mesa, can alter the polarization, thus giving a significant $E_{z}$ component.

\section{DEPOLARIZATION FIELD}

It is well known that the screening of the light field by a high-density electron gas leads to an upward shift in the energy of the intersubband transition. In a parabolic band approximation, ${ }^{22}$ the line shape is unaltered but the absorption occurs at an energy $\tilde{E}$ where

$$
\tilde{E}^{2}=E_{12}^{2}+E_{\mathrm{pl}}^{2} ;
$$

$E_{\mathrm{pl}}$ is a plasma energy given by

$$
E_{\mathrm{pl}}^{2}=\frac{2 e^{2} N_{s} S E_{12}}{\varepsilon_{0} \varepsilon_{r}}
$$

and $S$ is the depolarization integral

$$
S=\int_{-\infty}^{\infty}\left[\int_{-\infty}^{z} \psi^{2}\left(z^{\prime}\right) \psi^{1}\left(z^{\prime}\right) d z^{\prime}\right]^{2} d z .
$$

What is less well known, however, is that the depolarization effect leads to a line narrowing for a nonparabolic system. ${ }^{23}$ The InAs/AlSb wells here provide an extreme example because of the high degree of nonparabolicity in the band structure of InAs and the high carrier concentrations. For instance, a $150-\AA$ InAs/AlSb well populated out to a Fermi wave vector of $k_{f}=0.025 \AA^{-1}\left(N_{s}=1 \times 10^{12} \mathrm{~cm}^{-2}\right)$ has an intersubband energy that is $122.9 \mathrm{meV}$ at $k_{x}=0$ but only $104.4 \mathrm{meV}$ at $k_{x}=k_{f}$. Owing to the small density of states in InAs we can safely neglect the exciton effect, which is important in the wider gap systems GaAs and $\mathrm{Si}$. 
To incorporate the depolarization effect into our theory we have extended the original time-dependent perturbation theory of Allen et al. ${ }^{22}$ to cope with a multiple-component wave function. For the real part of the conductivity $\sigma_{z z}$, we arrive at

$$
\operatorname{Re}\left(\sigma_{z z}\right)=\frac{2 e^{2}}{\hbar} \frac{N_{s}\left\langle z_{12}\right\rangle^{2} E}{E_{12}(0)} \mathfrak{I}\left(\frac{G(E)}{1+\left(E_{\mathrm{pl}}^{2} / E_{12}(0)^{2}\right) G(E)}\right)
$$

with

$$
G(E)=\frac{1}{\pi N_{s}} \int_{0}^{k_{f}} \frac{k E_{12}(0)^{2}}{E_{12}(k)^{2}-E^{2}-i E \Gamma} d k,
$$

in agreement with Załużny. ${ }^{23}$ In these equations, $E$ is the light energy, and $\Gamma$ is an energy broadening of the levels. ${ }^{24}$ For an infinitely high quantum well in a one-band model the $S$ integral can be calculated analytically to give $S=5 L / 9 \pi^{2}, 8.44 \AA$ for $L=150 \AA$. A calculation of $S$ using the multiple-component wave functions yields the remarkably similar $8.49 \AA$, and furthermore exhibits practically no $k_{x}$ dependence. This is another indication of the independence of spatial matrix elements on band nonparabolicity. In contrast to Załużny, ${ }^{23}$ who considered a GaAs system with, therefore, modest nonparabolicity, our model calculations of Fig. 6 reveal the full extent of the line narrowing through the depolarization field. We plot the line shape for $S=0$ (i.e., without the depolarization) and for $S \neq 0$ for $\Gamma=1$ and 5 $\mathrm{meV}$. It can be seen very clearly in the, unfortunately, as yet unrealizable case $\Gamma=1 \mathrm{meV}$ that the main peak in $\operatorname{Re}\left(\sigma_{z z}\right)$ has a width determined not by the spread of the function $G(E)$ but simply by the line broadening $\Gamma$. Additionally, there is a weak absorption in the one-particle energy range, which evolves into a low-energy tail at higher $\Gamma$. The integrated absorption is the same in both cases. The energy of the peak, however, does depend on the spread of the oneparticle energies, i.e., on the carrier density and on the exact band structure, and is difficult to estimate without computing the integrals in Eq. (13).

The experimental data of Fig. 5 for the $150-\AA$ well verify that the depolarization field is playing an important role in that the measured energy, $134 \mathrm{meV}$, is substantially higher than even the calculated $k_{x}=0$ single-particle gap, 122.9 meV. Furthermore, the linewidth, $6.6 \mathrm{meV}$, is considerably narrower than the broadening expected from nonparabolicity alone.

To make a further comparison of the calculated and measured energies we have the problem that the carrier concentration in the $150-\AA$ sample is high enough for the second subband to be occupied, although, curiously, we have not seen any experimental evidence that this is the case. We make the approximation that the first subband is filled so that the Fermi energy lies just below the second subband. This corresponds to a density of $1.8 \times 10^{12} \mathrm{~cm}^{-2}$ for the $150-\AA$ well. Including the depolarization field and taking $n=1.8 \times 10^{12} \mathrm{~cm}^{-2}$ we calculate an intersubband energy of $132.7 \mathrm{meV}$, which is in excellent agreement with the experiment. (We cannot expect better agreement than $\sim 10 \mathrm{meV}$, as we have neglected both band bending and strain, which make small contributions to the intersubband energy.) For the 100- $\AA$ well, the increased 1-2 energy implies that the
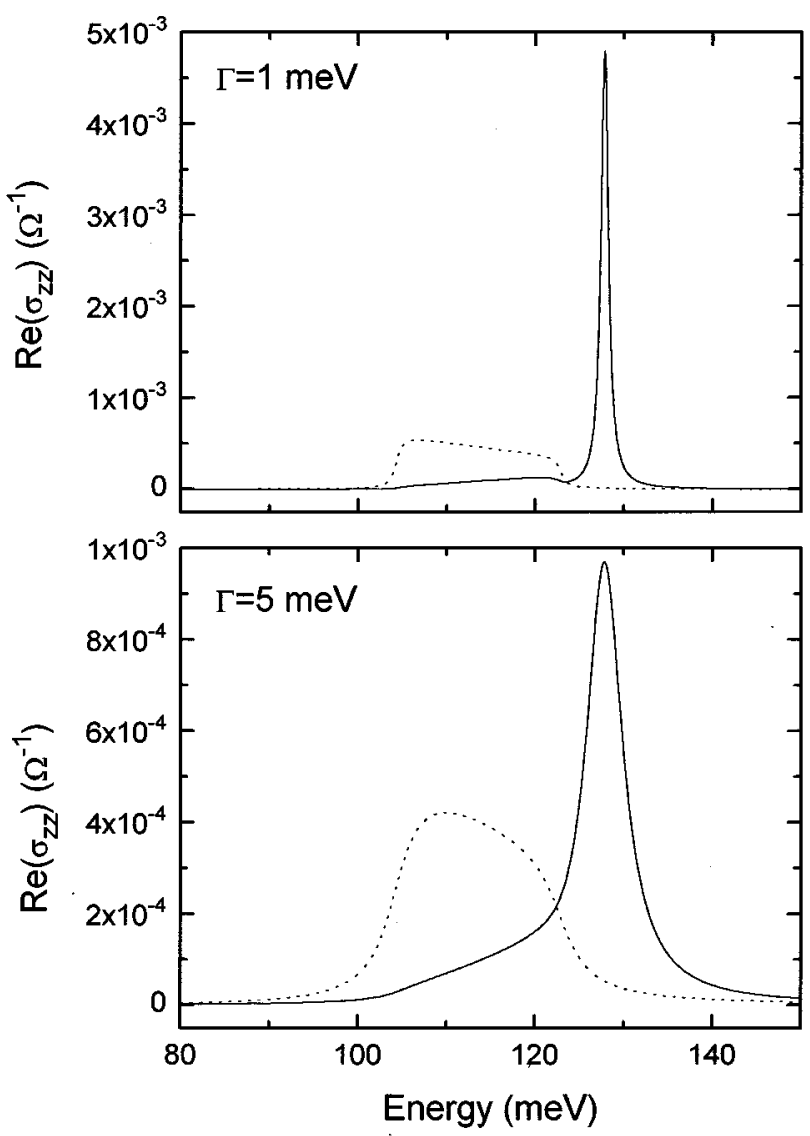

FIG. 6. The calculated real part of the conductivity for a $150-\AA$ quantum well with carrier concentration $1 \times 10^{12} \mathrm{~cm}^{-2} . S=0$ (dotted lines) neglects the depolarization field; $S \neq 0$ (solid lines) includes it, and results are given for values of the broadening parameter $\Gamma, 1$ and $5 \mathrm{meV}$.

second subband cannot be conceivably occupied at low temperature, and the theory with $n=3.0 \times 10^{12} \mathrm{~cm}^{-2}$ predicts $213 \mathrm{meV}$ as against the measured $248 \mathrm{meV}$. The origin of this discrepancy is not clear at present.

The absorption $A$ is related to the conductivity by

$$
A=\frac{\operatorname{Re}\left(\sigma_{z z}\right)}{\varepsilon_{0} c \sqrt{\varepsilon_{r}}}
$$

which allows a direct comparison of the experimentally measured spectrum with the theoretically calculated conductivity. We have used Eq. (15) with an approximation for the electric field distribution ${ }^{21}$ to estimate experimentally the matrix element $\left\langle z_{12}\right\rangle$. We find $\left\langle z_{12}\right\rangle=28 \pm 5$ and $\langle z\rangle=23 \pm 4 \AA$ for the 150 - and 100- $\AA$ samples, respectively, as compared to the calculated 31.0 and $21.9 \AA$. In fact, a more precise way of measuring $\left\langle z_{12}\right\rangle$ is to excite the intersubband resonance with an in-plane magnetic field and light polarized in the plane, ${ }^{25}$ thus avoiding the complications of the field distribution in the Brewster angle geometry. With this technique, we find the similar $\left\langle z_{12}\right\rangle=25.0 \AA$ for the 150 - $\AA$ well.

\section{CONCLUSIONS}

The one-band picture of intersubband resonance has come under attack recently because nonparabolicity implies sub- 
stantial $p$-type wave-function admixture in the eigenfunctions, which is obviously neglected in the simple picture. We have presented here both calculations and experiments pertinent to InAs/AlSb quantum wells where nonparabolicity effects, as judged by band-structure measurements such as cyclotron resonance, are known to be particularly large. Intersubband resonance can be described by essentially three properties, the energy, the matrix element $\left\langle z_{12}\right\rangle$, and the line shape. We find that only the energy of the transition is strongly influenced by the nonparabolicity. The one-band estimate of $\left\langle z_{12}\right\rangle$ differs only by $\sim 10 \%$ as compared to a multiband calculation in the $\mathbf{k} \cdot \mathbf{p}$ approximation. The oneband picture predicts that other matrix elements corresponding to $1-2_{x}, 1-3_{x}$, and $1-3_{z}$ are rigorously zero. In the multiband picture this is not correct, but we find that the 1-3 matrix elements are negligibly small, and that $1-2_{x}$ is at most $\sim 1 \%$ of $1-2_{z}$. Furthermore, we show that the nonparabolicity broadening does not strongly influence the line shape because of the very pronounced effect of the depolarization field. A comparison of measured intersubband absorption with our theory gives most satisfactory agreement.
The results on the line narrowing are important in a device context, as a broad response would be undesirable for detectors and lasers based on the intersubband transition. The calculations also show that, for a given intersubband energy and carrier concentration, the matrix element $\left\langle z_{12}\right\rangle$ should be made as large as possible to achieve the maximum absorption. This immediately implies that a narrow-gap material offers significant advantages over GaAs in that for a given energy the well can be much wider, leading to a larger $\left\langle z_{12}\right\rangle$.

\section{ACKNOWLEDGMENTS}

The work in Munich was sponsored by the Volkswagen Stiftung. The Santa Barbara group would like to acknowledge support from the Office of Naval Research, QUEST, and the NSF Science and Technology Center for Quantized Electronic Structures (Grant No. DMR 91-20007). R.J.W. would like to thank both the Alexander von Humboldt Stiftung and the EEC for financial support.
${ }^{1}$ R. Q. Yang, J. M. Xu, and M. Sweeny, Phys. Rev. B 50, 7474 (1994).

${ }^{2}$ A. Shik, in Intersubband Transitions in Quantum Wells, edited by E. Rosencher et al. (Plenum Press, New York, 1992), p. 319.

${ }^{3}$ L. H. Peng and C. G. Fonstad, J. Appl. Phys. 77, 747 (1995).

${ }^{4}$ L. H. Peng and C. G. Fonstad, Appl. Phys. Lett. 62, 3342 (1993); L. H. Peng, J. H. Smet, T. P. E. Broekaert, and C. G. Fonstad, ibid. 61, 2078 (1992).

${ }^{5}$ H. S. Li, R. P. G. Karunasiri, Y. W. Chen, and K. L. Wang, J. Vac. Sci. Technol. B 11 (3), 922 (1993).

${ }^{6}$ Z. Yuan, Z. Chen, D. Cui, J. Ma, Q. Hu, J. Zhou, and Y. Zhou, Appl. Phys. Lett. 67, 930 (1995).

${ }^{7}$ C. Gauer, J. Scriba, A. Wixforth, J. P. Kotthaus, C. R. Bolognesi, C. Nguyen, B. Brar, and H. Kroemer, Semicond. Sci. Technol. 9, 1580 (1994).

${ }^{8}$ E. O. Kane, J. Phys. Chem. Solids 1, 249 (1957).

${ }^{9}$ The boundary conditions are those that are conventionally used in envelope function calculations, but they have no compelling experimental justification for this material system. We do not attempt to explore this issue here.

${ }^{10}$ L. M. Roth, B. Lax, and S. Zwerdling, Phys. Rev. 114, 90 (1959).

${ }^{11}$ M. H. Weiler, in Semiconductors and Semimetals, edited by R. K. Willardson and A. C. Beer (Academic, New York, 1981), Vol. 16, p. 119.
${ }^{12}$ K. Suzuki and J. C. Hensel, Phys. Rev. B 9, 4184 (1974).

${ }^{13}$ J. Khurgin, Appl. Phys. Lett. 62, 1390 (1993).

${ }^{14}$ Y. Takada, K. Aral, N. Uchimura, and Y. Uemura, J. Phys. Soc. Jpn. 49, 1851 (1980); Y. Takada, ibid. 50, 1998 (1981).

${ }^{15}$ W. Zawadzki, J. Phys. C 16, 229 (1983).

${ }^{16}$ W. Beinvogl and J. F. Koch, Solid State Commun. 24, 687 (1977).

${ }^{17}$ K. Wiesinger, H. Reisinger, and F. Koch, Surf. Sci. 113, 102 (1982).

${ }^{18}$ H. Reisinger and F. Koch, Solid State Commun. 37, 429 (1981).

${ }^{19}$ G. E. Marques and L. J. Sham, Surf. Sci. 113, 131 (1982).

${ }^{20}$ R. Q. Yang and J. M. Xu, Phys. Rev. B 46, 6969 (1992).

${ }^{21}$ F. Capasso, C. Sirtori, D. Sivco, and A. Y. Cho, in Intersubband Transitions in Quantum Wells, edited by E. Rosencher et al. (Plenum Press, New York, 1992), p. 141.

${ }^{22}$ S. J. Allen, Jr., D. C. Tsui, and B. Vinter, Solid State Commun. 20, 425 (1976).

${ }^{23}$ M. Załużny, Phys. Rev. B 43, 4511 (1991).

${ }^{24}$ The experimental spectra of Fig. 5 are actually closer to Gaussians than to Lorentzians, and this is one point of departure between the theory and the measurements.

${ }^{25}$ C. Gauer, A. Wixforth, J. P. Kotthaus, M. Kubisa, W. Zawadzki, B. Brar, and H. Kroemer, Phys. Rev. Lett. 74, 2772 (1995); C. Gauer, A. Wixforth, J. P. Kotthaus, G. Abstreiter, and G. Weimann, Europhys. Lett. 30, 111 (1995). 